\title{
Integralidade na Atenção Primária: análise do discurso acerca da organização da oferta do teste rápido anti-HIV
}

\author{
The integrality in Primary Healthcare: discourse analysis about the organization of the provision of the \\ rapid anti-HIV test
}

Integralidad en Atención Primaria: análisis del discurso sobre la organización de la oferta de la prueba rápida del VIH

Haline Costa dos Santos Guedes ${ }^{1}$ (1) José Nildo de Barros Silva Júnior² (B)

Glaydes Nely Sousa da Silva ${ }^{1}$ (B) Débora Raquel Soares Guedes Trigueiro ${ }^{1}$ (D) Jordana de Almeida Nogueira² ${ }^{2}$ Anne Jaquelyne Roque Barrêto² (1)

1. Faculdade de Enfermagem Nova Esperança. João Pessoa, PB, Brasil.

2. Universidade Federal da Paraíba, Programa de Pós-graduação em Enfermagem. João Pessoa, PB, Brasil.
Autor correspondente:

José Nildo de Barros Silva Júnior

E-mail: nildoenfer@hotmail.com

Recebido em 16/01/2020.

Aprovado em 18/06/2020.

\section{RESUMO}

Objetivo: Analisar o discurso dos gerentes saúde relacionado à organização da oferta do teste rápido anti-HIV, na perspectiva do atributo integralidade. Método: Pesquisa exploratória de natureza qualitativa realizada em um distrito sanitário do município de João Pessoa/PB. A coleta de dados foi realizada em setembro de 2017, utilizando-se um roteiro de entrevista semiestruturado, o qual foi aplicado a treze gerentes de saúde de serviços de Atenção Primária à Saúde. O material empírico foi analisado à luz do dispositivo teórico-metodológico de Análise de Discurso, identificando-se o bloco discursivo: Organização dos serviços para a realização do teste rápido anti-HIV. Resultados: Os discursos apontam a filiação ideológica que compreende a fragmentação da assistência, normatização da demanda com enfoque em agendamentos, estigmatização e desresponsabilização do cuidado, dificultando a realização da integralidade na perspectiva da descentralização da oferta do teste rápido anti-HIV. Conclusão e implicações para a prática: $O$ discurso dos gerentes de saúde aponta fragilidades na qualidade do serviço e organização da oferta do teste rápido anti-HIV. Executar as políticas para reverter a situação atual e a educação permanente dos profissionais podem potencializar a integralidade do serviço.

Palavras-chave: Atenção Primária à Saúde; HIV; Diagnóstico Precoce; Infecções por HIV; Integralidade em Saúde.

\section{ABSTRACT}

Objective: Analyze health managers discourse related to the organization of the rapid anti-HIV test provision, from the perspective of the integrality attribute. Method: Exploratory research of qualitative nature, undertaken in a health district of the municipality of João Pessoa, Paraíba. Data collection was carried out in September 2017, using a semi-structured interview script for a semi-structured interview, which was applied to thirteen health care service managers from Primary Health Care services. The empirical material was analyzed in the light of the theoretical-methodological Discourse Analysis device, identifying the discursive block: Organization of services for conducting the rapid anti-HIV test. Results: The speeches point out to the ideological affiliation that comprises the fragmentation of the assistance, standardization of the demand with focus on schedules, stigmatization and unaccountability of the care, making it difficult to achieve comprehensiveness in the perspective of the decentralization of the offer of rapid anti-HIV test. Conclusion and implications for practice: The discourse of health managers points out weaknesses in the quality of service and organization of the rapid anti- HIV test provision. Implementing policies to reverse the current situation and continuing education for professionals can enhance the integrality of the service.

Keywords: Primary Health Care; HIV; Early Diagnosis; HIV Infections; Integrality in Health.

\section{RESUMEN}

Objetivo: Analizar el discurso de los gerentes de la salud relacionados con la organización de la oferta de pruebas rápidas anti-VIH, desde la perspectiva del atributo de integralidad. Método: Investigación exploratoria de naturaleza cualitativa, realizada en un distrito sanitario del municipio de João Pessoa/PB. La recogida de datos se llevó a cabo en septiembre de 2017, utilizando un plan de entrevista semiestructurada, que se aplicó a trece gerentes de la salud de servicios de Atención Primaria de la Salud. El material empírico se analizó a la luz del dispositivo metodológico teórico del Análisis del Discurso, identificando el bloqueo discursivo: Organización de servicios para realizar la prueba rápida anti-VIH. Resultados: Los discursos señalan la afiliación ideológica que incluye la fragmentación de la asistencia, la normatización de la demanda con enfoque en los horarios, la estigmatización y la irresponsabilidad de la atención, lo que dificulta alcanzar la integralidad desde la perspectiva de la descentralización la oferta de prueba rápida anti-VIH. Conclusión e implicaciones para la práctica: El discurso de los gerentes de la salud señala las deficiencias en la calidad del servicio y la organización de la oferta de pruebas rápidas anti -VIH. La implementación de políticas para revertir la situación actual y la educación permanente de los profesionales puede potenciar la integralidad del servicio.

Palabras clave: Atención Primaria de Salud; VIH; Diagnóstico Precoz; Infecciones por VIH; Integralidad en Salud. 


\section{INTRODUÇÃO}

Sabe-se, atualmente, que há avanços no que concerne ao enfrentamento do Vírus da Imunodeficiência Humana (HIV), porém, nem todo usuário tem acesso equitativo. Foi visto que, ao passar do tempo, tal infecção sempre esteve permeada por condutas e práticas estigmatizadas, distanciando o usuário do diagnóstico precoce e fragilizando as políticas de saúde desenvolvidas em resposta ao HIV. ${ }^{1}$

Segundo The Joint United Nations Programme on HIV/Aids (UNAIDS), em 2018, havia 37,9 milhões de casos de HIV no mundo, sendo 36,2 milhões em adultos e 1,7 milhão em crianças menores de quinze anos. ${ }^{2}$

No Brasil, em 2018, foram notificados pelo Sinan 43.941 casos de infecção pelo HIV.Destes, de acordo com o sexo, foram 207.207 (69,0\%) de homens e 93.220 (31,0\%) de mulheres. A região com maior concentração é a Sudeste, seguida da Nordeste e Sul com, $16.586(37,7 \%), 10.808(24,6 \%)$ e $7.838(17,8 \%)$ respectivamente, seguidas da região Norte com 5.084 (11,6\%) e 3.625 (8,2\%) na região Centro-Oeste. Sabe-se que a subnotificação compulsória do HIV dificulta uma melhor avaliação dos casos de infecção no Brasil e interfere, diretamente, na efetivação de políticas públicas para melhor gestão do cuidado a esse agravo. ${ }^{3}$

A eclosão do HIV/Aids no Brasil foi um marco concomitante no confronto do declínio político, econômico e social, caracterizado pela mudança da condução democrática brasileira e pelo desencadeamento e reestruturação de inúmeras mobilizações sociais, nas quais o governo brasileiro vem implementando ações e serviços com o objetivo de diminuir a taxa de incidência da aids no país. ${ }^{4}$

Dentre estas ações e serviços, destaca-se a descentralização do Teste Rápido (TR) anti-HIV como uma resposta imediata a demanda de casos de HIV/Aids, além da ampliação do acesso e a formação do vínculo entre profissional e usuário, visto que a Atenção Primária à Saúde (APS) é preconizada como a porta de entrada do Sistema Único de Saúde (SUS), sendo consolidado como eixo ordenador, no qual essa inserção de oferta de diagnóstico expressou uma nova face das políticas de saúde, desprendendo-se do processo centralizado para a consolidação de um modelo descentralizado. ${ }^{5}$

A APS é operacionalizada através de quatro atributos que visam a discussão da qualidade da atenção e sua organização, apresentando, dentre estes, o atributo integralidade como característica definidora do serviço da APS e princípio-diretriz do SUS, o qual visa possibilitar a organização da oferta do TR anti-HIV e garantir a permeabilidade em todos os níveis do sistema de saúde, conforme necessidade do usuário. ${ }^{6,7}$

Dessa forma, a integralidade é interpretada através de uma construção coletiva, pois apresenta sentidos representados pela 'a integralidade como um traço da boa medicina', 'a integralidade como um princípio/modo de organizar as práticas de saúde' e a 'integralidade como respostas governamentais a problemas específicos de saúde'. O presente estudo fará abordagem a partir da integralidade como modo de organizar as práticas de saúde, que pode ser compreendida como a necessidade da horizontalidade e maior alcance dos programas e políticas de saúde, bem como inclusão de assistência e práticas preventivas, na perspectiva de superar a fragmentação das praxes nas unidades de saúde. ${ }^{7}$

Em 2004, o Ministério da Saúde (MS) legitimou como método de controle e prevenção da infecção o TR anti-HIV na APS para abranger com mais eficácia os atores sociais que se encontram em vulnerabilidade frente à infecção, potencializando a proposta através da portaria no 29, de 17 de dezembro de 2013, o que favorece a mudança das ações no controle do HIV, além de aproximar a oferta do TR anti-HIV ao usuário. ${ }^{8,9}$

Copiosas são as problematizações na APS, impasses que dificultam a realização da descentralização do TR anti-HIV, o que exige inclusão gradativa dessa descentralização, compreendida como uma ação de difícil incorporação na APS atribuída ao estigma da doença, oferta do teste no período gestacional e a fragmentação da atenção, que, por consequência, comprometem a qualidade dos serviços de saúde em referência ao manejo da infecção pautada no atributo integralidade, para concessão de parâmetros que auxiliam na tomada de decisão acerca das políticas vigentes. ${ }^{8,10}$

O entendimento da problemática descrita apresenta a proposta deste estudo, que surgiu pela necessidade de compreender o processo de descentralização do TR nos serviços de APS a partir do discurso dos gerentes saúde responsáveis pela condução da implementação das políticas e programas de saúde e, consequentemente, analisar a organização da APS e sua influência na garantia da integralidade a respeito da oferta do TR anti-HIV.

Não foram identificados estudos que versem acerca da organização dos serviços de saúde na APS para TR anti-HIV, sobretudo, tendo os discursos dos gerentes saúde como foco da investigação. Dessa maneira, questiona-se: como os gerentes saúde das equipes de serviços de APS organizam a oferta do TR anti-HIV? Objetivando-se, neste estudo, analisar o discurso dos gerentes saúde relacionado à organização da oferta do TR anti-HIV, na perspectiva do atributo integralidade.

\section{MÉTODO}

Pesquisa exploratória de natureza qualitativa realizado junto aos Gerentes Saúde dos serviços de APS, em Distrito Sanitário de um município localizado na região leste do estado da Paraíba. O referido Distrito Sanitário foi escolhido por abranger e concentrar o maior número de usuários que acessam o TR anti-HIV.

O Programa Gerente Saúde respalda-se pela Política Nacional de Atenção Básica (PNAB), estabelecida como norma pelo MS e a Organização Mundial de Saúde, tornando-se referência para todos os municípios brasileiros. ${ }^{11}$

Como critério de inclusão dos sujeitos, considerou-se a situação de atuar como gestor no sistema local de saúde, representados na figura dos gerentes saúde, excluindo-se da pesquisa os gerentes saúde que estivessem afastados do seu serviço, sendo aplicada a técnica de saturação teórica, na qual à medida que as entrevistas eram realizadas, desempenhava-se 
um processo contínuo de análise dos dados, de forma individual, identificada pelo distanciamento de novos elementos nos discursos.

Foram entrevistados treze profissionais dos referidos serviços, dentre os quais quatro fisioterapeutas, quatro assistentes sociais, duas fonoaudiólogas, dois profissionais de educação física e um administrador.

A coleta de dados foi realizada em setembro de 2017, em ambiente escolhido por eles, sendo todas as entrevistas realizadas nos serviços de atuação do gerente participante do estudo. As entrevistas foram realizadas nos turnos da manhã e tarde, individualmente, em sala reservada, mediante a assinatura do Consentimento Informado. Foi utilizado um roteiro de entrevista semiestruturado, com questões relacionadas ao objeto de estudo.

Para obter o material empírico, as entrevistas foram gravadas por meio de um smartphone, com duração média de 20 minutos, sendo, posteriormente, transcritas na íntegra. Com a finalidade de preservar a imagem dos sujeitos colaboradores, os nomes foram substituídos pela sigla $\mathrm{G}$ alusivo ao nome gerente, enumerando-a na sequência das entrevistas, ou seja, G1 a G13.

No intuito de expor o caráter do contexto histórico, mantendo como elemento em comum a centralidade no discurso e destacando a possível observação do mesmo a partir de diferentes perspectivas, foi utilizado o dispositivo teórico-metodológico da Análise de Discurso (AD) de linha francesa. ${ }^{12}$

A AD francesa interpreta a linguagem como prática social, fundamentada por um sujeito do inconsciente, ressaltando que ela não busca resposta certa ou errada, mas procura expor o que é e como funciona. ${ }^{13}$ Levando em conta que as práticas do pensamento são expostas através dos discursos, reserva-se à $A D$ francesa a função de elucidar o processo de construção através da língua.

$\mathrm{Na}$ análise dos dados, a etapa inicial acontece à circunscrição do conceito-análise, tendo, como escopo da análise, a saturação determinada pela recorrência do discurso a ponto de ser encerrado. Nesse instante, será fomentando o corpus. Após a definição do corpus, acontece a leitura flutuante feita sem ênfase significativa e, em seguida, a leitura analítica para ajudar o analista a evidenciar os sentidos que respondam às três perguntas heurísticas: 1. Qual é o conceito-análise presente no texto? 2. Como o texto constrói o conceito-análise? 3. A que discurso pertence o conceito-análise construído da forma que o texto constrói? ${ }^{13}$

Para a interpretação do corpus do estudo foi utilizado o conceito-análise "Organização da oferta do teste rápido antiHIV'. Em seguida, buscou-se identificar os sentidos atribuídos pelos gerentes saúde acerca da organização dos serviços de APS para a oferta do TR anti-HIV por meio de leituras exaustivas, identificação de marcas textuais até haver a saturação de sentidos. Por conseguinte, buscou-se localizar o sentido construído pelos gerentes saúde acerca do objeto de estudo, evidenciando o funcionamento da ideologia na textualização.

A segunda etapa é definida pela escrita da análise: a caracterização da análise através da contextualização e explicação do tema a partir do que ele será tratado; explicitação do dispositivo teórico-analítico. ${ }^{13}$ Dessa forma, foi possível a identificação do seguinte bloco discursivo: Organização do serviço de saúde para a realização do TR anti-HIV.

O projeto de pesquisa foi enviado ao Comitê de Ética em Pesquisa-CEP da Faculdade de Enfermagem e de Medicina Nova Esperança-FACENE/FAMENE, sob nํ이 de protocolo 108/2017 e CAAE no: 72757817.6.0000.5179, contemplando as orientações legais e éticas, respeitando o protocolo de pesquisa envolvendo seres humanos, contidos na resolução 466/2012 CNS/MS.

\section{RESULTADOS}

O bloco discursivo Organização do serviço de saúde para a realização do TR anti-HIV foi formado por meio de enunciados e respectivos fragmentos discursivos dos gerentes saúde relativos à organização da oferta do TR anti-HIV na APS, a seguir apresentados (Quadro 1).

\section{DISCUSSÃO}

Através dos fragmentos, foram vistas situações que afetam a integralidade da assistência, fragilizando a organização da oferta do TR anti-HIV quanto a: organização da APS, acessibilidade, capacitação do profissional de saúde, transferência de responsabilidade, dispositivos organizacionais, estigmatização, falta de familiaridade a respeito da integralidade e articulações frágeis.

Assim, o escopo da realização da análise discursiva foi a organização da oferta do TR anti-HIV por meio do atributo integralidade. Sabe-se que a integralidade é compreendida como uma construção coletiva e polissêmica em seus sentidos, os discursos possibilitaram a identificação da integralidade princípio/modo de organizar as práticas de saúde, emergindo a necessidade da horizontalidade para efetivar o preconizado pelo MS a respeito da oferta do TR anti-HIV. ${ }^{14}$

A gestão local reconhece que o TR anti-HIV é uma atribuição da APS, descentralizando o fluxo do diagnóstico do Centro de Testagem e Acolhimento (CTA) e do âmbito de referência para tratamento, cumprindo o preconizado pelas diretrizes organizacionais do SUS. Dessa maneira, garantindo o fluxo organizacional, esse segmento torna-se imprescindível para a concretização da realização do TR anti-HIV no município em estudo.

Os discursos expõem uma situação que potencializa a procura do usuário pelo serviço especializado para diagnóstico e tratamento, uma vez que as políticas atuais de enfrentamento ao HIV introduzem uma proposta de descentralização das ações de controle do HIV na APS no sentido de ampliar a permeabilidade aos serviços de saúde, de maneira que favoreça o enfrentamento e controle do HIV. ${ }^{15}$ Os sentidos atribuídos aos discursos dos gerentes saúde remetem que os usuários deveriam se dirigir a serviços especializados para a obtenção do diagnóstico, talvez, pelo fato de estas unidades contarem com profissionais capacitados e estrutura adequada, para atender aos usuários 
Quadro 1. Recorte discursivo dos gerentes saúde em relação à organização dos serviços na oferta do TR anti-HIV na APS. João Pessoa, PB, Brasil, 2017.

\begin{tabular}{|c|c|}
\hline Enunciado & Fragmentos discursivos \\
\hline $\begin{array}{l}\text { Organização da APS na oferta } \\
\text { do TR anti-HIV }\end{array}$ & $\begin{array}{l}\text { Isso era para ser de conhecimento da equipe, infelizmente isso eu falo de maneira geral, } \\
\text { não só daqui eu mesma me coloco dentro dessas pessoas, a gente desconhece muito o } \\
\text { fluxo do município [...]. (G13) }\end{array}$ \\
\hline Acessibilidade ao TR anti-HIV & $\begin{array}{l}\text { Aqui na unidade não temos o teste rápido, porque nós não temos esses profissionais [...] } \\
\text { (G1) }\end{array}$ \\
\hline $\begin{array}{l}\text { Capacitação do profissional } \\
\text { de saúde para a oferta do TR } \\
\text { anti-HIV }\end{array}$ & $\begin{array}{l}\text { Aqui na unidade não tem teste rápido, porque só pode fazer o teste rápido as pessoas que } \\
\text { tem a capacitação para [...] nenhum aqui tem a capacitação do teste rápido, na verdade, } \\
\text { nenhum deles se interessaram [...]. (G6) } \\
\text { [...]nós não temos ainda os profissionais capacitados, [...] a gente tem profissionais de } \\
\text { outra equipe de saúde que eles vêm dá o suporte quando são realizadas essas ações } \\
\text { esporadicamente [...]. (G8) }\end{array}$ \\
\hline $\begin{array}{l}\text { Transferência de } \\
\text { responsabilidade para } \\
\text { execução do TR anti-HIV }\end{array}$ & $\begin{array}{l}\text { [...] ela encaminha para a enfermeira da unidade que faz, que é a que tem ela faz, já com } \\
\text { o resultado ele é encaminhado para a equipe dele de referência, junto com a médica e a } \\
\text { enfermeira, eles dão o encaminhamento. (G2) } \\
\text { [...] ] la [enfermeira] vai fazer a notificação e encaminhar para receber o coquetel. (G9) } \\
\text { [...]quando ela [enfermeira] identifica ela encaminha para o Centro de Testagem e } \\
\text { Aconselhamento (CTA) [...]. (G10) }\end{array}$ \\
\hline $\begin{array}{l}\text { Distanciamento da } \\
\text { Longitudinalidade }\end{array}$ & $\begin{array}{l}\text { [...] a nossa unidade na segunda é marcada o agendamento para a sexta feira, para os } \\
\text { usuários vir e fazer na sexta. (G7) }\end{array}$ \\
\hline Dispositivos organizacionais & $\begin{array}{l}\text { [...] Antes a gente fazia agendado e quando agenda eles faltavam, agora está sendo } \\
\text { demanda livre, [...] tem em sala de espera, tem ações, em ações é muito solicitado. (G5) } \\
\text { São feitas as atividades de prevenção, voltado e estimulado sempre em atendimento em } \\
\text { sala de espera [...] O enfrentamento é feito nesse sentido também na busca ativa dos } \\
\text { pacientes que tem e procuram o serviço [...], [...]é agendado o dia e as vezes também } \\
\text { demanda espontânea, é ofertado nessas unidades que são referências [...] (G12) }\end{array}$ \\
\hline $\begin{array}{l}\text { Estigmatização de pessoas } \\
\text { vivendo com HIV/Aids }\end{array}$ & $\begin{array}{l}\text { [...] g gente tacha como soropositivo, a gente só tacha como uma pessoa que tem HIV } \\
\text { e eu acredito que esse tipo de usuário eu o encaminho para o Clementino (hospital de } \\
\text { referência) para o tratamento [...] (G13) }\end{array}$ \\
\hline $\begin{array}{l}\text { Falta de familiaridade a } \\
\text { respeito da integralidade na } \\
\text { oferta do TR anti-HIV }\end{array}$ & $\begin{array}{l}\text { [...] elas fazem com as gestantes que já faz parte dos exames que elas fazem de rotina, } \\
\text { como também elas fazem com os ditos grupos de risco [...] com essa questão das gestantes } \\
\text { (G3) }\end{array}$ \\
\hline $\begin{array}{l}\text { Fragilidade na articulação } \\
\text { entre os pontos de atenção }\end{array}$ & $\begin{array}{l}\text { [...]ele já sabe para onde encaminhar o enfermeiro ou médico já o direciona para um local } \\
\text { específico de tratamento [...]. (G4) } \\
\text { Aqui não é ofertado, se fosse a gente encaminharia. (G6) } \\
\text { [...]ela vai fazer a notificação e encaminhar para receber o coquetel. (G9) } \\
\text { [...]ele foi encaminhado para o Clementino (hospital de referência), [...]a parte dos } \\
\text { coquetéis é todo encaminhado para lá [...], faz o exame se deu positivo a gente encaminha } \\
\text { direto para lá... (G11) }\end{array}$ \\
\hline
\end{tabular}

e, concomitantemente, estar fora da competência da APS em virtude da falta de profissional qualificado ou oferta do exame.

Os gerentes relatam que não executam o TR devido aos profissionais não se interessarem pela capacitação, impossibilitando a realização do exame na APS, o qual torna o usuário susceptível ao diagnóstico tardio, prejudicando a operacionalização do TR anti-HIV, além de ressaltar a importância da presença da gestão na implementação do teste, com o propósito de acolher a demanda dos adscritos, com ênfase na privacidade e ética. ${ }^{16}$
Seguindo a linha de interpretação dos sentidos, os gerentes saúde atribuem ao outro, neste caso, aos profissionais de saúde, a falta de interesse na qualificação para a oferta do TR anti-HIV na APS. Nessa perspectiva, há indícios de que os discursos apontam a desresponsabilização dos profissionais na oferta do exame.

Os discursos revelam a transferência de responsabilidade de identificar e referenciar usuários com diagnóstico positivo de HIV para o enfermeiro. A sequência discursiva "ela [enfermeira] vai fazer a notificação"; "ela [enfermeira] identifica [..] e encaminha 
para o CTA" terceiriza a responsabilidade apenas a um profissional, o que sinaliza fragmentação do cuidado.

Observa-se que o atendimento está centralizado na assistência especializada. A fragmentação do cuidado está relacionada à desarticulação da comunicação intersetorial, sabendo que é importante a articulação entre os três níveis, necessitando da efetivação das Redes de Atenção à Saúde (RAS) que representa um problema no contexto nacional e internacional. Além disso a centralização da assistência representa a descontinuidade e contribui para a inaptidão profissional, favorecendo a fragmentação do cuidado e distanciando-o de uma atuação resolutiva. ${ }^{17,18}$

Em outros enunciados, os gerentes saúde revelam sentidos que indicam barreiras para a realização do TR anti-HIV nos serviços de APS, quando apresentam marcas textuais como "não é feito nesta unidade", "só podem fazer o teste rápido as pessoas que têm capacitação" e "já o direciona para um local específico" favorece a busca pelos serviços especializados, indo de contramão à política de descentralização do diagnóstico do HIV na APS.

Nesse sentido, os resultados aqui apresentados não refletem apenas uma realidade local, pois o estudo realizado na APS em oito Unidades Básicas de Saúde da Família (UBSF) também identificou barreiras para realizar o TR nas unidades de saúde, destacando a inexistência de qualquer interesse na criação de vínculo usuário/profissional, e menos ainda, na qualidade do atendimento. Em outro estudo realizado, foi visto que na opinião dos usuários entrevistados, as relações dos profissionais são de caráter não vinculativo, ou seja, há ausência de resolutividade, atraso de nomeação, consulta e espera na fila. ${ }^{19}$

A partir da sequência discursiva "na nossa unidade, na segunda é marcado o agendamento para [...] os usuários virem e fazerem na sexta" produz sentidos indiretos da dificuldade dos usuários para a realização do exame e fragmentos como "elas [a enfermeira] fazem com as gestantes que já faz parte dos exames [...] de rotina"; "como também elas [enfermeiras] fazem com os ditos grupos de risco" é revelado uma organização de serviço para TR anti-HIV dirigidos à limitação da oferta especificada pela demanda de grupo de risco e atendimento específico, apontando barreiras que fragilizam a integralidade, devido ao distanciamento da criação de vínculo e acolhimento necessários para ofertar o TR anti-HIV.

No discurso de G3 o ponto central da oferta do TR anti-HIV é direcionado para as gestantes, excluindo-se os demais segmentos populacionais. Ao entrevistar as equipes foi revelada a delimitação do público apenas para as gestantes, pois o TR já faz parte da rotina de exames solicitados no pré-natal. ${ }^{5}$ Por outro lado, a barreira na oferta de TR anti-HIV para os demais indivíduos da comunidade está atrelada à sexualidade e moralidade associada ao HIV. São, além disso, observados pontos de estrangulamento para a realização do TR anti-HIV nas unidades pesquisadas entretanto, o estudo revela que a aceitação para realizar o exame é alta, ressaltando que a disponibilização do TR na APS otimiza o acesso, necessitando apenas de organização da oferta. ${ }^{20}$
Ainda sobre o discurso, sem perceber, os gerentes saúde evidenciaram por meio da memória discursiva, sentidos e significados que apontam para uma organização de serviço que fere a longitudinalidade, denominada continuidade do cuidado. Dessa forma, contribui para a criação de confiança, laços interpessoais entre profissionais e usuários, os quais favoreçam a oferta e a realização do teste. E, nessa lógica, em caso de positividade do exame para o HIV, o usuário necessita permear o serviço, além de evitar encaminhamentos desnecessários para os serviços especializados, fazendo com que a longitudinalidade contribua para a criação de corresponsabilização entre profissionais e usuários ao longo do tempo. ${ }^{21,22}$

A longitudinalidade potencializa o atributo integralidade, tendo em vista o diagnóstico precoce e preciso com o objetivo de iniciar a Terapia Antirretroviral (TARV), como visto em estudo feito na África do Sul, no qual a TARV é ofertada na APS e prescrita por enfermeiros profissionais em gestão do HIV, para a diminuição da carga viral na circulação sanguínea, o que, consequentemente, diminui a propagação do vírus. ${ }^{23}$

Emergiram nos discursos dispositivos organizacionais que otimizam a oferta do TR fortalecendo o atributo integralidade, tais como: sala de espera e busca ativa, conforme as sequências discursivas - "são feitas as atividades de prevenção [...] em atendimento em sala de espera, [...] busca ativa dos pacientes que têm e procuram o serviço". Dessa forma, a sala de espera constitui-se de um espaço otimizador para os usuários relatarem as vivências do seu cotidiano, refletirem sobre o processo saúde-doença e serem sensibilizados a realizar a testagem anti-HIV. ${ }^{24}$

A busca ativa presente no discurso de G12 faz alusão aos pacientes que procuram a unidade, ou seja, demanda espontânea, no qual é aguçada a marca textual no discurso construído pela memória dos sentidos "[...]é agendado o dia e, às vezes, também demanda espontânea, é ofertado nessas unidades que são referências[...]". Este arranjo organizacional se refere ao atendimento não programado, pois contempla apenas necessidades momentâneas, remetendo a um processo laboral voltado para uma assistência biomédica, cujas intervenções são concretizadas apenas quando o usuário procura a unidade de saúde. Sabe-se que o modelo assistencial, onde as ações organizacionais são permeadas pela demanda espontânea, com escopo na cura, ocasiona um distanciamento do preconizado pelo modelo vigente, que é a prevenção e promoção à saúde. ${ }^{25}$

O fragmento discursivo por meio das marcas textuais "é agendado o dia, e às vezes demanda espontânea" potencializa o distanciamento do acolhimento considerado uma ferramenta importantíssima para a criação de um vínculo com o usuário, propiciando laços interpessoais que refletem a reciprocidade entre os profissionais e usuários, no intuito de repensar a assistência em saúde e fortalecer a integralidade, de modo que o usuário se sinta acolhido e seguro para fazer o TR anti-HIV. ${ }^{21}$

Pensando na fragmentação supracitada, um estudo aponta a integralidade como chave da amostra, trazendo à tona que tal atributo é abrangente e transcende o acesso aos pontos da 
rede, que para garantia da longevidade do usuário no serviço de saúde, é necessário haver um compromisso com a qualidade da assistência prestada. Desse modo, contemplar a integralidade na APS é um desafio, cabendo aos profissionais compreenderem a realidade local para a criação de novas intervenções e romper com esse modelo centrado na doença, o qual ainda prevalece nesse nível de atenção, buscando na promoção, proteção e recuperação da saúde. ${ }^{26,27}$

Surgiu nas segmentações de G13 um discurso atrelado à historicidade do HIV, aguçada nas marcas textuais "a gente tacha como soropositivo", "a gente só tacha como uma pessoa que tem HIV" "eu acredito que esse tipo de usuário". Tal discurso é justificado como memória discursiva em alusão à estigmatização de pessoas vivendo com HIV/Aids recuperada por discursos já mencionados, renovando a historicidade do sucedido discursivo, sendo materializada pela lembrança social estabelecida na historicidade..$^{28}$

Foram vistos em estudo o estigma e a discriminação associados ao HIV/Aids, referido pelos usuários nos serviços da APS, sendo que os profissionais reconhecem o próprio preconceito, e enaltecem esse mesmo sentimento dos próprios usuários. ${ }^{28}$ Este fato pode ser compreendido por sua historicidade, pois antigamente, as pessoas com HIV eram rotuladas pelo seu grupo de risco, como a orientação sexual, ou pelo comportamento de risco como o uso de drogas ou profissionais do sexo. Atualmente, mesmo com a evolução do enfrentamento da infecção, a estigmatização ainda está presente na sociedade e entre profissionais da saúde, havendo relatos de estudos em que indivíduos com o vírus receberam recusa na assistência ao revelar sua condição de doença. ${ }^{29}$

A partir do discurso de G3 e G13, fica notório que, no atual contexto, a integralidade está fora de alcance, sendo predominantemente voltada para as gestantes, deixando sem assistência os demais adscritos, o que potencializa a fragmentação da assistência. O MS preconiza uma assistência no nível primário de atenção equânime e coletiva com expectativa da atenção holística, visto que a integralidade é um fator primordial que favorece $a$ assistência contínua às pessoas vivendo com HIV/Aids..$^{30}$

Apesar de a descentralização do TR anti-HIV na APS se constituir como uma política a ser implementada nos serviços, os fragmentos discursivos revelam marcas textuais como "ele já sabe para onde encaminhar", além de "algum usuário que tenha algum sintoma", "até caso de abuso sexual essas coisas", "referência para o Clementino (hospital de referência)", "ela vai fazer a notificação e encaminhar para receber o coquetel". Observa-se na oratória o distanciamento do usuário em efetivar o exame, realizando-o apenas através de aspectos clínicos. $O$ usuário, automaticamente, é direcionado para o serviço de referência hospitalar, sendo resgatada em sua memória a hegemonia hospitalocêntrica, cujos cuidados são baseados apenas em sintomas já instalados, norteando-se em cuidados hospitalares, ao invés da utilização da APS como nível preventivo. Ainda nesses discursos, evidencia-se que o gerente saúde não tem familiaridade a respeito do percurso do usuário nas Redes de Atenção à Saúde (RAS). ${ }^{28}$

A integralidade perpassa os demais níveis da assistência, no entanto, é na APS que se encontra a sua maior significância estratégica, devido à resolutividade e necessidade de resposta aos usuários, à família e comunidade. ${ }^{22}$ Assim, o direcionamento do manejo do diagnóstico da infecção para a APS ocorreu pela possibilidade de maior permeabilidade e continuidade dos cuidados com qualidade, uma vez que é responsável por coordenar e ordenar o cuidado em sua área de abrangência. ${ }^{20}$

As RAS foram designadas pelo MS com a perspectiva de reestruturar e, principalmente, superar o modelo fragmentado de gerenciamento e assistência à saúde, na pretensão de assegurar as ações que os usuários necessitam. As RAS são contempladas como um sistema de múltiplos serviços de saúde, procurando ofertar uma atenção integral e contínua aos adscritos, devendo ser coordenadas através da APS por meio das linhas de cuidado. ${ }^{31}$

Compreende-se, portanto, que a organização do serviço para a oferta do TR anti-HIV no nível primário de atenção não converge com o atributo integralidade na medida em que há distanciamento dos discursos dos gerentes em relação ao preconizado pelas diretrizes do SUS. Nesta perspectiva, o discurso que sustenta os sentidos da organização da oferta de TR anti-HIV em serviços de APS, a partir dos gerentes saúde, é o discurso hegemônico, com característica biomédica e hospitalocêntrica, uma vez que o escopo está na oferta do teste para as gestantes e no encaminhamento dos usuários para os serviços hospitalares especializados ou de referência do município a fim de obterem a detecção da infecção pelo HIV.

\section{CONCLUSÃO E IMPLICAÇÕES PARA A PRÁTICA}

A partir da delimitação representacional do TR anti-HIV neste estudo, observou-se que os discursos apontam fragilidades na qualidade do serviço quanto à organização e o exercício do atributo integralidade referente à oferta do teste, permeada por barreiras basilares na gestão do cuidado como a não apropriação de sua posição como agente potencializador na detecção e notificação de casos, além da carência do aporte regular do paciente na APS e seu uso contínuo ao longo do tempo, na perspectiva da implementação das políticas de saúde concernente ao HIV/Aids. Essa condição requer uma apreensão e execução das políticas, de modo a reverter a situação atual do agravo.

Destaca-se, como principal limitação, a restrição do sujeito de pesquisa ao gerente saúde, deixando de lado outros profissionais que atuam diretamente na oferta do TR anti-HIV a exemplo de enfermeiros e médicos, elucidando apenas a experiência de um profissional e não de todos que estão na produção de cuidado às pessoas em exposição ao HIV. Entretanto, elucidar os discursos dos gerentes saúde é estratégico para compreender a organização da oferta de TR anti-HIV nos serviços de APS, uma vez que este profissional desempenha funções administrativas 
capazes de modificar uma dada realidade, tanto na perspectiva de reestruturação do serviço de saúde quanto da qualificação de recursos humanos.

Assim, verificamos a partir do material analisado, que os discursos apontam para a filiação ideológica que compreende a fragmentação da assistência, normatização da demanda com foco em agendamentos, estigmatização e desresponsabilização do cuidado, dificultando a realização da integralidade com ênfase na descentralização da oferta do TR anti-HIV.

Por isso, sugere-se a produção de outros estudos que compreendam a inclusão dos gestores saúde e também de outros participantes como profissionais e usuários, uma vez que esta situação atual remete a necessidade de capacitação, maiores incentivos institucionais e políticos relacionados à educação permanente no cotidiano dos profissionais como instrumento de mudança nas práticas sociais, a fim de potencializar a integralidade do serviço e executar as políticas para reverter a situação atual do agravo.

\section{CONTRIBUIÇÕES DOS AUTORES}

Desenho do estudo. Aquisição, análise de dados e interpretação dos resultados. Redação e revisão crítica do manuscrito. Aprovação da versão final do artigo. Responsabilidade por todos os aspectos do conteúdo e a integridade do artigo publicado. Haline Costa dos Santos Guedes. José Nildo de Barros Silva Júnior.

Análise de dados e interpretação dos resultados. Redação e revisão crítica do manuscrito. Aprovação da versão final do artigo. Responsabilidade por todos os aspectos do conteúdo e da integridade do artigo publicado. Glaydes Nely Sousa da Silva.

Desenho do estudo. Análise de dados e interpretação dos resultados. Redação e revisão crítica do manuscrito. Aprovação da versão final do artigo. Responsabilidade por todos os aspectos do conteúdo e a integridade do artigo publicado Débora Raquel Soares Guedes Trigueiro. Anne Jaquelyne Roque Barrêto.

Análise e interpretação dos resultados e revisão crítica do manuscrito. Aprovação da versão final do artigo. Responsabilidade por todos os aspectos do conteúdo e a integridade do artigo publicado. Jordana de Almeida Nogueira.

\section{EDITOR ASSOCIADO}

Maria Catarina Salvador da Motta.

\section{REFERÊNCIAS}

1. Trigueiro DRSG, Almeida AS, Monroe AA, Costa GPO, Bezerra VP, Nogueira JA. AIDS and jail: social representations of women in freedom deprivation situations. Rev Esc Enferm USP. 2016 jun/ago;50(4):554-61. http://dx.doi.org/10.1590/S0080-623420160000500003. PMid:27680039

2. United Nations Programme on HIV/AIDS. Estatísticas globais sobre HIV 2019 [Internet]. Brasília: Ministério da Saúde; 2019 [citado 2019 out 8]. Disponível em: https://unaids.org.br/estatisticas/

3. Ministério da Saúde (BR). Secretaria de Vigilância em Saúde. Departamento de Doenças de Condições Crônicas e Infecções Sexualmente Transmissíveis. Boletim Epidemiológico de HIV e Aids [Internet]. Brasília (DF): Ministério da Saúde; 2019 [citado 2019 dez
2]. Disponível em: http://www.aids.gov.br/pt-br/pub/2019/boletimepidemiologico-de-hivaids-2019

4. Rocha KB, Santos RRG, Conz J, Silveira ACT. Transversalizando a rede: O matriciamento na descentralização do aconselhamento e teste rápido para HIV, sífilis e hepatites. Saúde Debate. 2016 abr/jun;40(109):22-33. http://dx.doi.org/10.1590/0103-1104201610902.

5. Zambenedetti G, Silva RAN. Descentralização da atenção em HIV-Aids para a atenção básica: tensões e potencialidades. Physis. 2016;26(3):785806. http://dx.doi.org/10.1590/s0103-73312016000300005.

6. Carnut L. Cuidado, integralidade e atenção primária: articulação essencial para refletir sobre o setor saúde no Brasil. Saúde Debate. 2017;41(115):1177-86. http://dx.doi.org/10.1590/0103-1104201711515.

7. Diniz SGDM, Damasceno SS, Coutinho SED, Toso BRGDO, Collet N Avaliação do atributo integralidade na atenção à saúde da criança. Rev Gaúcha Enferm. 2016 dez;37(4):e57067. http://dx.doi.org/10.1590/19831447.2016.04.57067. PMid:27992031.

8. Arantes EO, Santos RS. Teste anti-HIV na perspectiva das políticas públicas: proposta e realidade. Rev Enferm UERJ. 2015 jun/ago;23(4):5626. http://dx.doi.org/10.12957/reueri.2015.16366.

9. Portaria $\mathrm{n}^{\circ} \mathbf{2 9}$, de 17 de dezembro de 2013 (BR). Aprova o manual técnico para o diagnóstico da infecção pelo HIV em adultos e crianças e dá outras providências. Diário Oficial da União [periódico na internet]. Brasília (DF): Ministério da Saúde; 2013 [citado 2019 out 23]. Disponível em: http://bvsms.saude.gov.br/bvs/saudelegis/svs/2013/ prt0029_17_12_2013.html

10. Lima JG, Giovanella L, Fausto MCR, Bousquat A, Silva EVD. Atributos essenciais da Atenção Primária à Saúde: resultados nacionais do PMAQ-AB. Saúde Debate. 2018 set;42(spe 1):52-66. http://dx.doi. org/10.1590/0103-11042018s104.

11. João Pessoa. Gerentes Saúde participam de reunião para avaliação de resultados na Secretaria Municipal de Saúde [Internet]. João Pessoa: Prefeitura Municipal; 2019 [citado 2019 out 29]. Disponível em: http:// www.joaopessoa.pb.gov.br/gerentes-saude-participam-de-reuniaopara-avaliacao-de-resultados-na-secretaria-municipal-de-saude/

12. Silva AR, Baptista DM. Abordagens de análise de discurso na ciência da informação: panorama dos estudos brasileiros. Inf Soc. [Internet]. 2015 maio/ago; [citado 2019 out 29];25(2):89-103. Disponível em: https://periodicos.ufpb.br/ojs2/index.php/ies/article/view/89

13. Souza SAF. Análise de discurso: procedimentos metodológicos. Manaus: Census; 2014

14. Silva MVS, Miranda GBN, Andrade MA. Sentidos atribuídos à integralidade: entre o que é preconizado e vivido na equipe multidisciplinar. Interface. 2017 jul/set;21(62):589-99. http://dx.doi.org/10.1590/1807-57622016.0420.

15. Norberg A, Nelson J, Holly C, Jewell ST, Lieggi M, Salmond S. Experiences of HIV-infected adults and healthcare providers with healthcare delivery practices that influence engagement in US primary healthcare settings: a qualitative systematic review. JBI Database System Rev Implement Rep. 2019 jun;17(6):1154-228. http://dx.doi. org/10.11124/JBISRIR-2017-003756.

16. Silva ITS, Valença CN, Silva RAR. Cartografia da implementação do teste rápido anti-HIV na Estratégia Saúde da Família: perspectiva de enfermeiros. Esc Anna Nery. 2017 set;21(4):1-8.

17. Santos AM, Almeida PF. Atención especializada en regiones de salud: desafíos para garantizar el cuidado integral en Brasil. Rev Salud Publica (Bogota). 2018 maio/jun;20(3):301-7. http://dx.doi.org/10.15446/rsap. v20n3.61392. PMid:30844001.

18. Medeiros RHA. Uma noção de matriciamento que merece ser resgatada para o encontro colaborativo entre equipes de saúde e serviços no SUS. Physis. 2015 out/dez;25(4):1165-84. http://dx.doi.org/10.1590/ S0103-73312015000400007.

19. Morais JMO, Morais FRR, Santiago CMC. First contact access in primary health care for children from 0 to 9 years old. R de Pesq: cuidado é fundamental Online. 2017;9(3):848-56. http://dx.doi.org/10.9789/21755361.rpcfo.v9.5575.

20. Araújo WJ, Quirino EMB, Pinho CM, Andrade MS. Percepção de enfermeiros executores de teste rápido em Unidades Básicas de Saúde. Rev Bras Enferm. 2018;71(Suppl 1):631-6. PMid:29562021. 
21. Paula CC, Silva CB, Nazário EG, Ferreira T, Schimith MD, Padoin SMM. Fatores que interferem no atributo longitudinalidade da atenção primária à saúde: revisão integrativa. Rev Eletrôn Enferm. 2015 out/ dez;17(4):1-11. http://dx.doi.org/10.5216/ree.v17i4.31084.

22. Gasparini MFV, Furtado JP. Longitudinalidade e integralidade no Programa Mais Médicos: um estudo avaliativo. Saúde Debate. 2019 jan/mar;43(120):30-42. http://dx.doi.org/10.1590/0103-1104201912002.

23. Solomons DJ, Merwe ASV, Esterhuizen TM, Crowley T. Factors influencing the confidence and knowledge of nurses prescribing antiretroviral treatment in a rural and urban district in the Western Cape province. South Afr J HIV Med. 2019 jul;20(1):1-7. http://dx.doi.org/10.4102/ sajhivmed.v20i1.923

24. Carrapato JFL, Castanheira ERL, Placideli N. Percepções dos profissionais de saúde da atenção primária sobre qualidade no processo de trabalho. Saude Soc. 2018 abr/jun;27(2):518-30. http://dx.doi.org/10.1590/ s0104-12902018170012.

25. Fertonani HP, Pires DE, Biff D, Scherer MDA. Modelo assistencial em saúde: conceitos e desafios para a atenção básica brasileira. Cien Saude Colet. 2015 jun;20(6):1869-78. http://dx.doi.org/10.1590/141381232015206.13272014. PMid:26060965.

26. Medeiros KKAS, Pinto Jr EP, Bousquat A, Medina MG. O desafio da integralidade no cuidado ao idoso, no âmbito da Atenção Primária à Saúde. Saúde Debate. 2017 set;41(3):288-95. http://dx.doi.org/10.1590/010311042017 s322.
27. Nakata LC, Feltrin AFS, Chaves LP, Ferreira JBB. Conceito de rede de atenção à saúde e suas características-chaves: uma revisão de escopo. Esc Anna Nery. 2020 jan;24(2):1-11. http://dx.doi.org/10.1590/21779465-ean-2019-0154.

28. Ew RAS, Ferreira GS, Moro LM, Rocha KB. Estigma e teste rápido na atenção básica: percepção de usuários e profissionais Rev Bras Promoc Saúde. 2018 jan/mar;31(3):1-11. http://dx.doi. org/10.5020/18061230.2018.7463.

29. Gabin CAS, Martins RF, Belila NM, Garbin AJI. O estigma de usuários do sistema público de saúde brasileiro em relação a indivíduos HIV positivo. DST-J Bras Doenças Sex Transm. [Internet]. 2017; [citado 2019 dez 18];29(1):12-16. Disponível em: https://pesquisa.bvsalud. org/portal/resource/pt/biblio-878798

30. Ministério da Saúde (BR), Secretaria de Vigilância em Saúde, Departamento de Vigilância. Prevenção e Controle das Infecções Sexualmente Transmissíveis, do HIV/Aids e das Hepatites Virais. Cuidado integral às pessoas que vivem com HIV pela Atenção Básica [Internet]. Brasília (DF): Ministério da Saúde; 2017 [citado 2019 dez 3]. Disponível em: http://bvsms.saude.gov.br/bvs/publicacoes/ cuidado_integral_hiv_manual_multiprofissional.pdf

31. Arruda C, Lopes SGR, Koerich MHAL, Winck DR, Meirelles BHS, Mello ALSF. Health care networks under the light of the complexity theory. Esc Anna Nery. 2015 jan/mar;19(1):169-73. http://dx. doi.org/10.5935/14148145.20150023. 